\title{
Correction to: Acoustic emission signal of fiber-reinforced composite grinding: frequency components and damage pattern recognition
}

Jinhua Wei ${ }^{1} \cdot$ Haoji Wang ${ }^{1} \cdot$ Bin $\operatorname{Lin}^{1,2} \cdot$ Tianyi Sui ${ }^{1} \cdot$ Feifei Zhao ${ }^{1}$. Sheng Fang ${ }^{2}$

Published online: 14 May 2019

(C) Springer-Verlag London Ltd., part of Springer Nature 2019

Correction to: The International Journal of Advanced Manufacturing Technology https://doi.org/10.1007/s00170-019-03645-x

The original version of this article contained a mistake.

The arrows and lines in Figs. 1(a) and Fig. 5 are missing.

The correct images are shown below.

The original article has been corrected.

The online version of the original article can be found at https://doi.org/ 10.1007/s00170-019-03645-x

Bin Lin

tdlinbin@126.com

Jinhua Wei

15222527210@163.com

Haoji Wang

18822408206@163.com

Tianyi Sui

tdsuity@163.com

Feifei Zhao

zhaofeifei760521@163.com

Sheng Fang

fangsheng0666@163.com

1 Key Laboratory of Advanced Ceramics and Machining Technology, Ministry of Education, Tianjin University, Tianjin, China

2 Key Laboratory of Advanced Functional Composites, CASC,

Beijing, China 
(a)

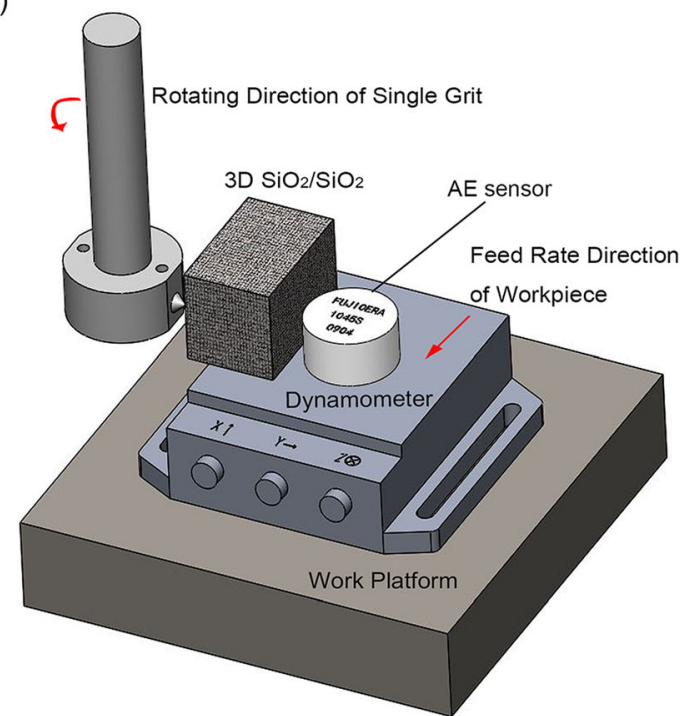

(b)

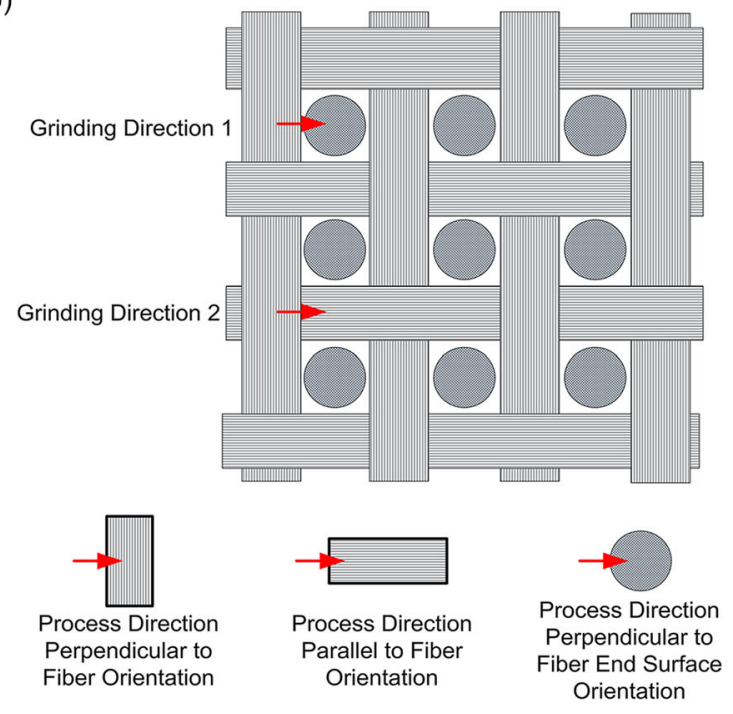

Fig. 1 Single-grain grinding experiment. a Experiment device setup. b Relation between fiber orientation and grinding direction
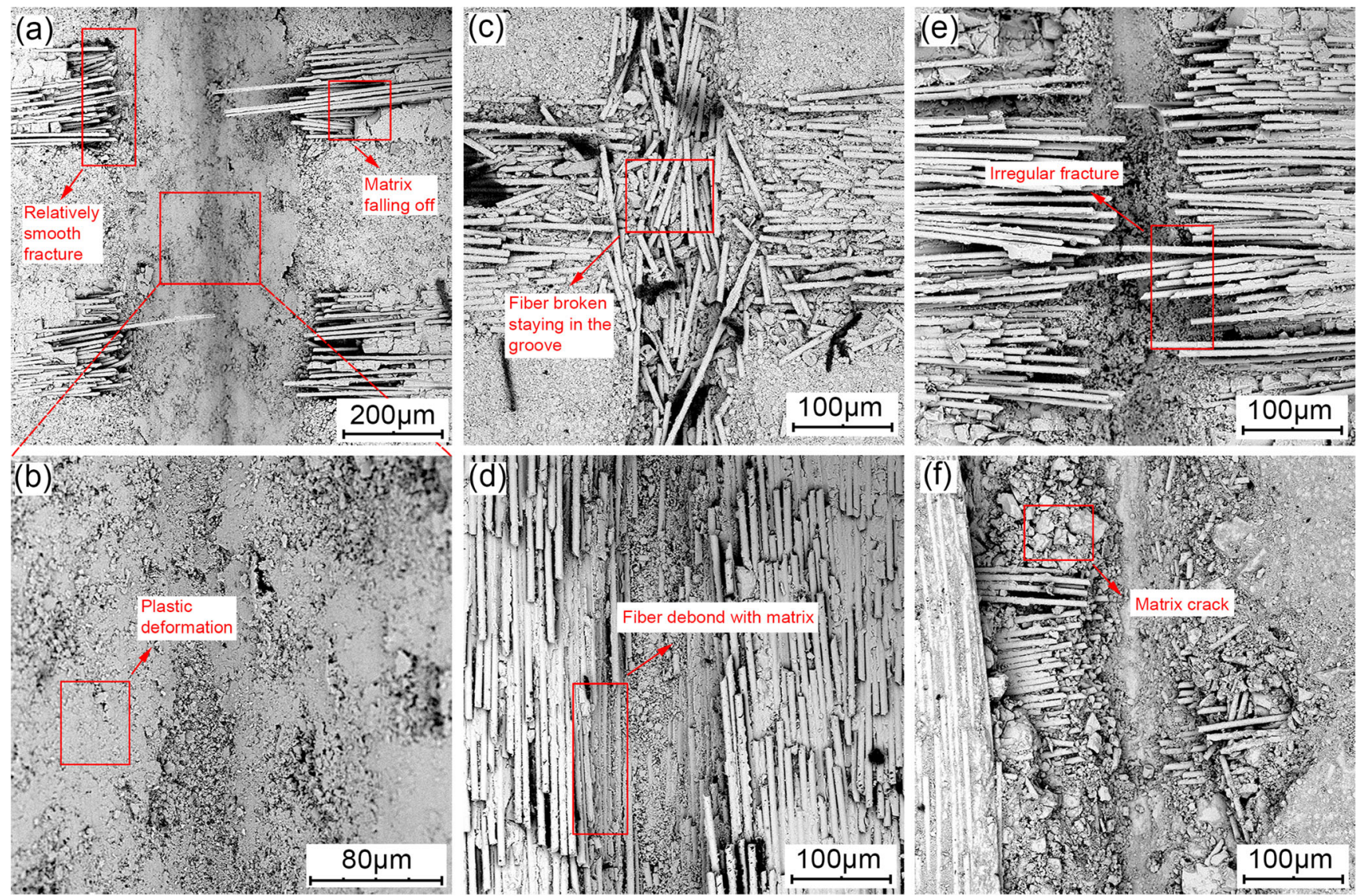

Fig. 5 SEM images of processed surfaces. a Processed surface on grinding direction 1. b Zoomed zone of grain vertex of (a). c, $\mathbf{d}$ fiber bundle side surface. e, f Processed surface on direction 2 with tool's heading direction perpendicular to fiber bundle side surface

Publisher's note Springer Nature remains neutral with regard to jurisdictional claims in published maps and institutional affiliations. 